\title{
The Occurrence, Properties and Significance of Citrinin Mycotoxin
}

\section{Doughari $\mathrm{JH}^{*}$}

Department of Microbiology, School of Pure and Applied Sciences, Modibbo Adama University of Technology, P.M.B 2076, Yola, Nigeria

\begin{abstract}
Citrinin is a nephrotoxic mycotoxin produced by several fungal strains belonging to the genera Penicillium, Aspergillus and Monascus. It contaminates various commodities of plant origin, cereals in particular, and is usually found together with another nephrotoxic mycotoxin, ochratoxin A. These two mycotoxins are believed to be involved in the etiology of endemic nephropathy. The mechanism of citrinin toxicity is not fully understood, especially not whether citrinin toxicity and genotoxicity are the consequence of oxidative stress or of increased permeability of mitochondrial membranes. Compared with other mycotoxins, citrinin contamination of food and feed is rather scarce. However it is reasonable to believe that humans are much more frequently exposed to citrinin than generally accepted, because it is produced by the same moulds as ochratoxin A which common contaminant of human food all over the world. Adequate knowledge of the toxin and proper food storage is essential to avoid contamination and further health and economic implication of citrinin poisoning.
\end{abstract}

Keywords: Cereals; Citrinin; Genotoxicity; Nephrotoxicity; Mycotoxins; Ochratoxin A

\section{Introduction}

Citrinin mycotoxin is a polyketide produced by several species of the genera Aspergillus, Penicillium and Monascus. Though A. niger is reported to be the highest producer of citrinin among the Aspergillus species, other citrinin producers of this genus include $A$. awentil, $A$. ostianus, A. fumigatus, A. niveus, A.awamori and A. parasiticus [1]. Some of the citrinin producing fungi are also able to produce the mycotoxins ochratoxin A or patulin [2]. Citrinin is generally formed after harvest under storage conditions and it occurs mainly in stored grains, but can also occur with other products of plant origin e.g. beans, fruits, fruit and vegetable juices, herbs and spices and also in spoiled dairy products [3].

In addition, citrinin is found as an undesirable contaminant in Red Mould Rice (RMR), which is used as a food preservative, and colourant in Asian foods [4]. The compound was shown to have broad antibacterial activity, thus prompting a growing interest and research for antibiotic agents in the middle of the last century. However, research interest decreased when the compound was demonstrated to have mammalian toxicity [2].

Storage of agricultural products has always been a challenge especially in Africa. Such agricultural stored produce usually is contaminated with a variety of fungi and pests. Consequently, the distribution of mycotoxins in many agricultural products is heterogeneous. It is therefore important that some of these toxins such as the citrinins and their health implications be adequately understood with a view to developing adequate preventive and control measures against food contamination. This review attempts to look at the nature and implications of citrinin in food crops [5].

\section{Natural Occurrence}

Citrinin is a mycotoxin originally isolated in 1931 by Hetherington and Raistrick from a culture of Penicillium citrinum. The toxin is produced worldwide in foodstuffs by microfungi of the genera, Penicillium and Monascus (Table 1) and a variety of other fungi that are found as contaminants of human foods, such as grain, cheese, sake, and red pigments as well as in spices $[3,6-8]$. The fungi that produce citrinin are major producers of other mycotoxins including ochratoxin
A and aflatoxins in grains. Consequently, co-occurrence of citrinin with ochratoxin A and aflatoxin B is common in grains, particularly rice. Simultaneous occurrence of the toxin with patulin in apple juices and apple jams has been reported [3].

Ostry et al. [6] also reported the occurrence of citrinin in a variety of foodstuffs of vegetable origin, e.g., cereals and cereal products, rice, pomaceous fruits (e.g., apples), fruit juices, black olive, roasted nuts (almonds, peanuts, hazelnuts, pistachio nuts), sunflower seeds, spices (e.g., turmeric, coriander, fennel, black pepper, cardamom and cumin) and food supplements based on rice fermented with red microfungi Monascus purpureus.. The European Food Safety Authority has also reported contamination of cheese by citrinin where toxigenic strains directly grow in the cheese mass [3].

Citrinin has also been found in commercial red yeast rice supplements. In so-called the "poisoning by moldy rice" case that occurred in Japan in 1953-54. Citrinin were found to be metabolite of the moulds Penicillium citrinum and Penicillium expansum, both postharvest pathogens of fruits (e.g., apple) and vegetables [7,9].

Literature data are scarce on natural occurrence of citrinin in indoor environments. However Tuomi et al. [10] did find citrinin in indoor materials; they analyzed 79 bulk samples of mouldy interior surfaces for 17 mycotoxins in buildings having moisture problems. The collected building materials included wallpaper, cardboard, wood, plasterboard, sand, soil, linoleum, polyurethane insulation, and paint. Three of the 79 samples were contaminated with citrinin. But also other mycotoxins such as sterigmatocystin, satratoxins, diacetoxyscirpenol, deoxynivalenol, verrucarol, and T-2-tetraol were present.

*Corresponding author: Doughari $\mathrm{JH}$, Department of Microbiology, School of Pure and Applied Sciences, Modibbo Adama University of Technology, P.M.B 2076 Yola, Nigeria, Tel: +234-7035599712; E-mail: jameshamuel@yahoo.com

Received December 01, 2015; Accepted December 10, 2015; Published December 15, 2015

Citation: Doughari JH (2015) The Occurrence, Properties and Significance of Citrinin Mycotoxin. J Plant Pathol Microbiol 6: 321. doi:10.4172/2157-7471.1000321

Copyright: ( 2015 Doughari JH. This is an open-access article distributed under the terms of the Creative Commons Attribution License, which permits unrestricted use, distribution, and reproduction in any medium, provided the original author and source are credited. 


\begin{tabular}{|c|c|c|c|c|}
\hline Genera & Subgenus & Series & Species & Foodstuff(examples) \\
\hline \multirow{4}{*}{ Penicillium } & Furcatum & ------ & P. citrinum & Cereals, nuts, fruit \\
\hline & Penicillium & Expansa & P. expansum & Fruit, cereals \\
\hline & Penicillium & Corymbifera & P. radicicola & Bulbs and root vegetables \\
\hline & Penicillium & Verrucosa & P. verrucosum & Cereals \\
\hline \multirow[t]{2}{*}{ Monascus } & M. purpureus & $\begin{array}{l}\text { Food supplement with fermented } \\
\text { red rice }\end{array}$ & & \\
\hline & M. ruber & Soya bean, sorghum, rice, oat & & \\
\hline
\end{tabular}

Table 1: Penicillium and Monascus species as citrinin producers in foodstuffs (source; Ostry et al., 2013).

\section{Physiology of Producer Cultures of Citrinin}

The major genera of fungi Penicillium, Aspergillus and Monascus spp associated with citrinin production are filamentous, and ubiquitous in the environment. Because they live a saprophytic mode of trophic life, they play very important role in decomposition processes of forest liter or dung, fruits or other organic materials [11]. Filamentous fungi when grown in culture, exhibit a high tendency towards spontaneous morphological or physiological change [12]. Penicillium spp with more than 150 different identified families are among the most commonly occurring worldwide and economically important members of the microfungi family. $P$. citrinun, the pioneer citrinin producing known occur in natural habitats such as air, soil, rhizosphere as well as the aqueous environment. The most important toxigenic species of Penicillium in foods include $P$. citreonigrum (which produces the toxin citreoviridin), $P$. expansum (citrinin), $P$. citrinum (produces citrinin), $P$. islandicum (cychlorotine, islanditoxin, erythroskyrin and luteoskyrin), P. vrrucosum (ochratoxin A, citrinin) and P. crustosum (penitrem A). In previous studies in which various toxigenic strains of Penicillium citrinum were investigated, although, minor genetic variations were observed, there was considerable uniformity of banding patterns among all the strains $[11,13,14]$. P citrinum is distinctively benicillus possessing a cluster of three to five divergent usually epically swollen metulae carrying long-columned conidia. Colonies, $25-30 \mathrm{~mm}$ on Czapek yeast extract agar (CYA) and $14-18 \mathrm{~mm}$ on meat extract agar (MEA) grow optimally at $37^{\circ} \mathrm{C}$ after 7 days of incubation.

Aspergillus spp, a largest aflatoxin producing fungal genus grows optimally on PDA, CYA, and MEA and produces black, small pale brown, to yellow green depending on the species. Diagnostic features include colour and texture of conidia, and the nature of toxin produced. Important mycotoxic species include $A$. flavus and $A$. parasiticus (produces aflatoxin A), A. ochraceus (ochratoxin A, citrinin, penicillin acid), A. versicolor (sterigmatocystin) $[15,16]$.

Monascus spp is also a genus comprising a wide range of fungi producing in addition to citrinin, a very wide range of useful colour pigment secondary metabolites including, monacolin $\mathrm{K}$, yellow monascin and ankaflavin, orange monascorubrin and rubropunktatin, red monascorubramin and rubropunktamin, monacolines, enzymes and lipids. Monascus spp the food fungi commonly called "red rice mold" have been consumed over the centuries in Asian countries as Monascus-fermented rice (MFR) locally called anka, beni koji and red yeast rice. It has been used traditionally as food colourant and preservative, food supplement and in traditional medicine [17]. The genus is characterized by rapid growth on red yeast rice extract agar (RYREA), MEA with colonies beginning as white, and then maturing into a pale pink, purple or grayish black colour depending on the species. On RYREA, the colonies are tapetum shaped with film shaped little or no wrinkled or radiation patterned skin membrane. The fungi acidophiles, with optimum $\mathrm{pH}$ and temperature of 3.55 .0 and $32-35^{\circ} \mathrm{C}$ and grows slowly on PDA $[6,18]$. The fungi are prototrophic, able to utilize ammonium and nitrate nitrogen sources and glucose and under anaerobic conditions can ferment glucose to ethanol with high yields [16].

\section{Biosynthesis and Genetics of Citrinin Biosynthesis}

The biosynthesis of citrinin in the genus Monascus appeared to be strain-specific and does not correlate with the pigments' biosynthesis by the fungal strains. The biosynthesis of the compound seem to originate from a tetraketide instead of a pentaketide as it was found in Aspergillus terreus and $P$. citrinum. Though both pigments and citrinin are derived from the same tetraketide, their synthesis is not reciprocally mutual or dependent upon the other. This independent level of production of each suggests that the enzymes involved in their synthesis have independent regulatory mechanisms of their genes. Consequently, a reduction in citrinin synthesis does not correlate with an increase in red pigments. Factors affecting production of these compounds and other secondary metabolites by filamentous microorganisms include respiration rate and hyphal morphology. Increase in respiration rate for instance makes oxygen transfer rate and $\mathrm{N}$ variables relevant to the process [19].

Strain improvement for increased citrinin and pigment biosynthesis has been reported by transformation with constructs of T-DNA inserted into strains of Monascus ruber using the vector Agrobacterium tumefaciens. Transformants were found to efficiently integrate the T-DNA gene into their genome and the transformed mutants were fully stable even after five successive cultures. Transformed mutants also demonstrated a greater citrinin and pigment production potential. Present molecular studies are based on transformation models targeting mainly functional genes for three important metabolites; pigment, citrinin and Monacolin K. Currently, the breeding of mutant transformant has been achieved by insertional inactivation in Monascus chromosomal genes. In addition, site-directed knockout technology has been developed for harmful citrinin synthase gene [17].

\section{Factors Affecting Citrinin Production}

Penicillium citrinum is one of the commonest microfungi on Earth, occurring in all kinds of food and feed, in almost all climates. Citrinin is produced over the range of $15-30^{\circ} \mathrm{C}$ and optimally at $30^{\circ} \mathrm{C}$. Factors such as a humidity of at least $16.5 \tilde{n} 19.5 \%$ favours the growth of the citrinin producing fungi on grain [20].

Fungal growth and mycotoxin production are also affected by the variety of agronomic practices and the nature of crops. In addition to weather conditions during harvest, postharvest, drying and cleaning, storage and processing conditions as well as toxigenic potential of the mould species also affect the toxin production [21].

\section{Decomposition of Citrinin}

Because citrinin is heat sensitive, it is unstable and therefore present in low levels in processed foods. In food processing, the compound decomposes during heat treatment to form other complex compounds, such as CIT H1 and CIT H2, whose cytotoxicity, compared to the 
original CIT, is higher and lower, respectively. This decomposition explains why [6].

Several studies have been carried out on degradation of citrinin revealing that decomposition of citrinin occurs at $>175^{\circ} \mathrm{C}$ under dry conditions, and at $>100^{\circ} \mathrm{C}$ in the presence of water. Known decomposition products include citrinin $\mathrm{H} 2$ which did not show significant cytotoxicity, while the decomposition product citrinin $\mathrm{H} 1$, which is made up of two citrinin molecules, showed an increase in cytotoxicity as compared to the parent compound [20,22]. Another decomposition product, the cytotoxic citrinin dimer, dicitrinin $\mathrm{A}$, was also reported in 2006, together with other monomeric and dimeric degradation products [23]

\section{Physical and Chemical Properties of Citrinin}

\section{Physical properties}

Citrinin has an appearance of solid lemon-yellow needles. Its solution changes colour in $\mathrm{pH}$, from lemon-yellow at $\mathrm{pH} 4.6$ to cherryred at $\mathrm{pH}$ 9.9. It has a melting point of $178.5^{\circ} \mathrm{C}$. The toxin is practically insoluble in cold water or sparingly soluble in hot water but soluble in aqueous sodium hydroxide, sodium carbonate, or sodium acetate; in methanol, acetonitrile, ethanol, and most other polar organic solvents $[2,20,24]$.

\section{Chemical properties and chemistry of citrinin}

Citrinin is a polyketide mycotoxin [C13H14O5, IUPAC: $(3 R$, 4S)-4,6-dihydro-8-hydroxy-3,4,5-trimethyl-6-oxo-3H-2-benzopyran7-carboxylic acid (Figure 1); molecular weight $250.25 \mathrm{~g} / \mathrm{mol}$; CAS No: 518-75-2]. It forms acidic lemon-yellow crystals with maximal ultraviolet (UV) absorption in methanol, melting at $175^{\circ} \mathrm{C}$ with decomposition. Citrinin crystallizes in a disordered structure, with the $p$-quinone and $o$-quinone tautomeric forms in a dynamic equilibrium in the solid state. It has a conjugated, planar structure which gives its natural fluorescence (the highest fluorescence is produced by a nonionized citrinin molecule at $\mathrm{pH} 2.5$ [25].

The toxin is capable of forming chelate complexes, and can be degraded in acidic or alkaline solution, or by heating. It is a quinone, with two intramolecular hydrogen bonds. Citrinin crystallizes in a disordered structure, with the $p$-quinone and $o$-quinone two tautomeric forms in a dynamic equilibrium in the solid state. In methanol or methanol/ methylene chloride mixtures, citrinin undergoes a Michaeltype nucleophilic addition reaction. This reaction is reversible, and the equilibrium shifts toward the normal citrinin if temperature is increased in methylene chloride [26].

In an investigation of a microbial fermentation of organic extract of Penicillium sp, Guangmin et al. were able to isolate three derivatives of citrinin. These compounds are; penicitrone A (also known as dicitrinin A), penicitrinol A, and penicitrinol B. [27]. Four new citrinin derivatives, including two citrinin dimers and two citrinin monomer derivatives, were isolated and identified from a marine-derived fungal strain Penicillium sp. along with six known related compounds. Their structures were elucidated by spectroscopic and chemical methods. The new compounds showed modest cytotoxic activity, and weak antimicrobial activity against Staphylococcus aureus. The isolated compounds are: two new citrinin dimers - penicitrinone $\mathrm{E}$, and penicitrinol J; and the monomers - penicitrinol $\mathrm{K}$, and citrinolactone D; citrinolactone B, citrinin, 2, 3, 4-trimethyl-5, 7-dihydroxy-2, 3-dihydrobenzofuran, and phenol A. [28].

\section{Laboratory Production of Citrinin}

Citrinin occurs naturally, and can also be obtained as an extract. Citrinin has mainly been found in rice, wheat, flour, barley, maize, rye, oats, peanuts and fruit and may co-occur in cereals with ochratoxin A. However, there is limited evidence of it surviving unchanged into cereal food products.

\section{Chemical synthesis}

The synthesis of citrinin was reported in 1949. Initially, the laevorotatory form of 3-(4,6-dihydroxy-ortho-tolyl)butan-2-ol is carboxylated to form the acid. This product is subjected to the Gattermann reaction (conversion of the phenol to the aromatic aldehyde by reaction with hydrogen cyanide/hydrogen chloride in the presence of a zinc chloride catalyst) to produce an intermediate, which is subsequently cyclized with sulphuric acid to form citrinin. The crude product was purified by crystallization from ethanol. An alternative synthetic method involves the conversion of dihydroxycitrinin to citrinin by oxidation with bromine [29].

In another experiment, culture supernatant $(10 \mathrm{l})$, was separated from the mycelia by centrifugation (Chilspin MSE Fisons, USA) at $4^{\circ} \mathrm{C}$ at $5000 \mathrm{rpm}$ for 15 minutes. The supernatant was the acidified to $\mathrm{pH} 5.0$ and extracted with ethyl acetate. The aqueous layer was removed and the organic layer was concentrated and applied to a silica gel 60 preparative TLC plate. The plates were examined under ultra violet light at $350 \mathrm{~nm}$ for the presence of a pale yellow spot $(\mathrm{R} f=0.6)$. The pale yellow active compound was removed from the plate and dissolved in methanol and again purified using HPLC (Hewlett-Packard 1090A, A Sphersorb C18, $5 \mu \mathrm{m}(25 \mathrm{~cm}$ by $4.6 \mathrm{~mm})$ column was eluted with methanol water $(20$ : $80, \mathrm{v} / \mathrm{v}$ ) at a flow rate of $1.0 \mathrm{ml} / \mathrm{min}$. the concentration of the citrinin was also measured in the culture supernatant spectrophotmetrically [30].

\section{Laboratory isolation}

Citrinin was first isolated in 1931 by Hetherington and Raistrick from a culture of Pennicillum citrinum Thom. A filtrate of the culture solution was acidified to precipitate the crude product; further purification was achieved by recrystallization from boiling absolute ethanol [20].

In the laboratory production process of citrinin, the PlackettBurman experimental design, a fractional factorial design, was used in order to demonstrate the relative importance of medium components on citrinin production and growth of $M$. ruber. Citrinin was produced from cultures of Monascus ruber by Abdulaziz and Moustafa in a submerged fermentation culture. Culture broth $(10 \mathrm{ml})$ was centrifuged in order to separate the fungal mycelium and the supernatant. The supernatant was concentrated 10 -fold and used for the determination

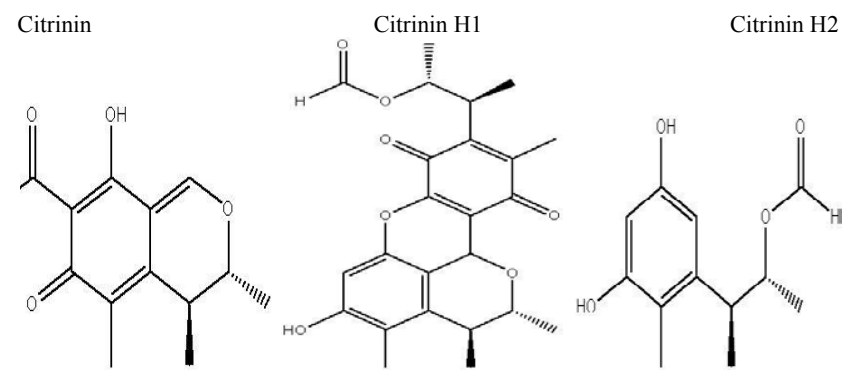

Figure 1: Chemical Structure of Citrinin, Citrinin $\mathrm{H} 1$, and Citrinin $\mathrm{H} 2$. 
of antibacterial activity. Thirty microliter $(30 \mu \mathrm{l})$ of culture supernatant were placed in each hole (6 $\mathrm{mm}$ of diameter) in Mueller-Hinton agar medium in a Petri-dish inoculated with $0.1 \mathrm{ml}$ bacterial suspension (3 $\times 106 \mathrm{cfu} / \mathrm{ml})$. Petri dishes were kept for $2 \mathrm{~h}$ in the refrigerator and then incubated for $12 \mathrm{~h}$ at $35^{\circ} \mathrm{C}$ and the inhibition zone diameter was then measured. The triplicate mean values obtained were considered the response [30].

For fungal biomass $(\mathrm{mg} / \mathrm{ml})$ determination, the sample culture after filtration through pre-weighed membrane filters $(45-\mu \mathrm{m}$ Millipore, Millipore Corp., Beford, Mass., USA), washed with sterile distilled water, and the mycelia then dried at $80^{\circ} \mathrm{C}$ to a constant weight [23]. Citrinin-producing fungi have been isolated on Potato Dextrose Agar (PDA) supplemented with antibiotics such as $0.005 \%$ chloramphenicol, grape juice agar (GJA) or yeast extract sucrose agar (YEA), [11,12]. Identification and characterization was based on estimation of viability, morphological appearance on agar, lactophenol cotton blue wet mount, random amplification of polymorphic DNA-polymerase chain reaction (RAPD-PCR) and fragment length polymorphism (AFLP) Santos, 2002; [11,13,14]. Further estimation and purification of citrinin levels have been achieved by various methods including thin layer chromatography (TLC) [11,12]. Other analytic methods for citrinin include colorimetric, fluorimetric, chromatographic techniques such as high performance liquid chromatography with fluorescence detection (HPLC-FLD), liquid chromatography mass spectrometry (LC-MS), gas chromatography mass spectrometry (GCMS) and immunochemical methods suchas EL:ISA (enzyme linked immunosorbent assay) [1,3,31].

\section{Pharmacological and Chemotherapeutic Potentials of Citrinin}

Citrinin is believed to be involved in the aetiology of endemic nephropathy. In addition to nephrotoxicity, Citrinin is also embryocidal and fetotoxic. The genotoxic properties of Citrinin have been demonstrated with the micronuleus test $(\mathrm{MN})$, but not with single-cell gel electrophoresis. The mechanism of citrinin toxicity is not fully understood, especially not whether citrinin toxicity and genotoxicity are the consequence of oxidative stress or of increased permeability of mitochondrial membranes. citrinin requires complex cellular biotransformation to exert mutagenicity [32].

\section{Antibacterial/antifungal potentials}

Citrinin has weak activity against Gram-positive bacteria, including Staphylococcus aureus, Bacillus subtilis, and Micrococcus luteus. It was almost or very ineffective against Gram-negative bacteria, yeasts, and molds. Interestingly, however, citrinin tended to inhibit the growth of some yeasts and molds in malt wort adjusted to acidic $\mathrm{pH}$.

The mycotoxin citrinin had antifungal activity under acidic conditions. At the minimum inhibitory concentration, it completely inhibited cellular respiration and partially inhibited the incorporation of radioactive precursors into macromolecules in Saccharomyces cerevisiae. It had no effect on cell permeability. In mitochondrial preparations, it significantly inhibited succinate oxidase and $\mathrm{NADH}$ oxidase. Rhizopus chinensis was more sensitive than S. cerevisiae; its growth and mycelial respiration at acidic $\mathrm{pH}$ were completely inhibited by lower concentrations of citrinin. The $\mathrm{pH}$-dependent antifungal activity of citrinin seems to be associated with its uptake by fungi. Approximately half of the citrinin taken up was found in mitochondria. The main site of the antifungal action of citrinin, therefore, appears to be the mitochondrial electron transport system [32].

\section{Anticancer potentials}

A variety of citrinin derivatives from different fungal species demonstrated antitumor potentials showing that Citrinin might be a precursor of novel active compounds against cancer disease [9].

Red yeast rice has been used in chinese medicine to strengthen the spleen, promote or improve digestion, eliminate dampness and phlegm, promote or improve blood circulation, and remove blood stasis. During the Ming Dynasty, red yeast rice was described as "sweet in flavor and warm in property." The genus Monascus has been used for centuries in Asia as a source of pigment for coloring traditional foods. The medicinal properties of red yeast rice are valued throughout Asia [33].

\section{Other Biotechnological Applications of Citrinin}

Red yeast rice (which contains citrinin) has been used to make rice wine and as a food preservative for maintaining the color and taste of fish and meat. Commercial food applications include coloration of sausage, hams, surimi, and tomato ketchup. The pigment has a long history of use as a food ingredient for Asian consumers, but not in Europe or America. However, a recent study documents the registration of numerous patents obtaining the use of Monascus as a food pigment in Japan, the United States, France, and Germany. Because citrinin is produced in by contaminating fungi, it has the potential of being developed into bio-weapon to be fed to hungry population of a wartorn country $[9,20]$.

\section{Health Implications of Citrinin Exposure}

Citrinin represents a severe problem especially in countries with a hot climate as under these conditions it is a major source of food poisoning after fungal contamination [34]. Citrinin (CIT), often found in the same food as ochratoxin A, is a powerful nephrotoxin [6]. In repeat dose toxicity studies, the kidney was identified as the principal target organ for CIT, and significant species differences in the susceptibility to CIT have been observed. The renal system of humans was found to be affected, and the mitochondrial respiratory chain was identified as a possible sensitive target for CIT. A few studies have also addressed its potential for immunotoxicity [35,36]. In animals and humans the toxin accumulates in the kidneys and can cause severe renal failure. Physiological investigations identified different adverse effects on the kidneys, liver and the gastrointestinal tract [33].

It has been suggested that citrinin may be implicated in the fatal human kidney disease, Balkan Endemic Nephropathy, along with other mycotoxins including ochratoxin A and further unidentified toxins. Citrinin can act synergistically with the ochratoxin A to attenuate the activity of RNA synthesis in kidney tissue.

Recently additive and synergistic nephrotoxic effects of citrinin in combination with other mycotoxins such as ochratoxin A have been described. The mycotoxin complex so formed, disrupted RNA synthesis in kidney tissue thereby further complicating its nephrotoxic potentials. Citrinin-Ochrotoxin A complex formation has been associated with alterations in renal function and/or with the development of renal pathologies. Simultaneous co-exposure to citrinin and ochratoxin A has also been reported to result in the modification of DNA adduct formation with increasing formation of the C-C8dG-OTA adduct [6]. The mycotoxin and ochratoxin complex A also are reported to be causative agents of hepatorenal carcinogenesis $[8,26]$.

Citrinin can be poisonous by ingestion and other routes, an experimental teratogen, other experimental reproductive effects 
a severe skin irritant, questionable carcinogen with experimental neoplastigenic and tumorigenic data and mutation data reported. When heated to decomposition it emits acrid smoke and irritating fumes.

Citrinin has been associated with yellow rice disease in Japan. It has also been implicated as a contributor to porcine nephropathy. Citrinin acts as a nephrotoxin in all animal species tested, but its acute toxicity varies in different species. The lethal dose for ducks is $57 \mathrm{mg} / \mathrm{kg}$; for chickens it is $95 \mathrm{mg} / \mathrm{kg}$; and for rabbits it is $134 \mathrm{mg} / \mathrm{kg}$. Citrinin can act synergistically with ochratoxin A to depress RNA synthesis in murine kidneys [37].

Exposure to mycotoxins through inhalation and skin contact can occur in indoor environments. However, the extent of possible health hazards caused by inhaled mycotoxins or through dermal exposure of mycotoxins is largely unclear [38].

\section{Control of Citrinin Contamination}

In order to control and prevent citrinin contamination, food containers should be tightly closed and kept in a well-ventilated place. Suitable protective clothing such as gloves, eye/face protection materials etc., should be worn at all times when coming in contact with items susceptible to citrinin contamination. In case of ingestion or contact with contaminated substances, it is appropriate to seek medical attention immediately.

\section{Conclusion}

The mycotoxin citrinin has both potentially important pharmacological applications as well as some significant medical implications. Therefore, extraction and purification of the metabolite and further toxicological studies will enable its appropriate understanding with a view to exploiting its usefulness, and controlling its harmful effects. Citrinin has been shown to produce serious illnesses in animals and even death. And its health implications to humans include irritation when contact is made in the eyes or skin.

There is a need for more data regarding the occurrence of citrinin in food and feed. There is a need for certified reference materials and defined performance criteria for the analysis of citrinin in food and feed. There is a need for well-designed toxicological studies in laboratory animal species to further explore the toxicological potential of citrinin and to characterize the dose-response relationships. There is a need for more data on farm animal toxicity and the carryover of citrinin from the feed to animal products intended for human consumption. There is also the need to public enlightenment and sensitization on the sources, effects and prevention of citrinin contamination.

\section{References}

1. Li YN, Wang YY, Zheng YQ, Guo YH (2010) Preparation and characterization of the high specificity monoclonal antibodies against citrinin. Progress in Biochemistry and Biophysics 37: 1248-1253.

2. Nejati P, Nosrati AC, Bayat M, Azar OL (2014) An investigation on measurement means of Citrinin toxin quantity by toxigenic Aspergillus species in biomass, using ELISA. International Journal of Advanced Biological and Biomedical Research 2: 2466-2471.

3. EFSA (2012) Scientific opinion on the risks for public and animal health related to the presence of citrinin in food and feed. EFSA Journal 10: 2605

4. Fink-Gremmels J, Dresel J, Leistner L (1991) Use of Monascus extracts as an alternative to nitrate in meat products. Fleischwirtschaft 71: 1184-1186.

5. Simpanya MF, Allotey J, Mpuchane S (2001) Insect and mycoflora interactions in maize flour. African Journal of Food and Nutritional Sciences 1:3-8.
6. Ostry V, Malir F, Ruprich J (2013) Producers and important dietary sources of ochratoxin A and citrinin. Toxins (Basel) 5: 1574-1586.

7. Wang ML, Lu CH, Xu QY, Song SY, Hu ZY, et al. (2013) Four new citrinin derivatives from a marine-derived Penicillium sp. fungal strain. Molecules 18 5723-5735.

8. Jeswal P, Kumar D (2015) Mycobiota and Natural Incidence of Aflatoxins, Ochratoxin A, and Citrinin in Indian Spices Confirmed by LC-MS/MS International Journal of Microbiology pp. 1-8.

9. Xu BJ, Gu LJ, Sung CK (2013) HPLC analysis of citrinin in red yeast rice. Food Science and Biotechnology 12: 376-380.

10. Tuomi T, Reijula K, Johnsson T, Hemminki K, Hintikka EL, et al. (2000) Mycotoxins in crude building materials from water-damaged buildings. App Environ Microbiol 66: 1899-1904.

11. Khosravi AR, Sheikhkaranf, M, Shokri H, Sabokbar A (2012) Genetic variability of citrinin-producingPenicillium citrinum strains as occupational health hazards in Northern Iran. Arh Hig Rada Toksikol 63: 489-496.

12. Santos IM, Abrunhosa L, Venâncio A, Lima N (2002) The effect of culture preservation techniques on patulin and citrinin production by Penicillium expansum Link. Lett Appl Microbiol 35: 272-275.

13. Abd-Elsalam KA, Omar MR, Migheli G, niremberg HI (2004) genetic characterization of Fosarium oxysporum $\mathrm{f}$. $\mathrm{sp}$. vasinfectum isolates by random amplification of polymorphic DNA (RPD) and amplified fragment length polymorphism (AFLP). Journal of Plant Disease Protection 111: 534-544.

14. El-Fadly GB, El-Kazzar MK, Hassan MAA, El-Kot GAN (2008) Identification of some Fusarium spp using RAPD-PCR Technique. Egypt Journal of Phytopathology 36: 71-80.

15. Pisareva E, Kujumdzieva A (2006) Taxonomic investigation and growth characteristics of citrinin free Monascus pilosus c1 strain. Biotechnology and Biotechnological Equipment 20: 1.

16. Pisareva E, Kujumdzieva A (2014) Characteristics of citrinin free Monascus pilosus C1 Strain. Biotechnology and Biotechnological Equipment 20: 88-96.

17. Srianta I, ristiarini S, Nugerahani I, Sen SK, zhang BB, et al. (2014) Recent research and development of Monascus ferementation products. International food Research journal 21: 1-12.

18. Wang TH, Lin TF (2007) Monascus rice products. Adv Food Nutr Res 53: 123-159.

19. Pisareva E, Savov V, Kujumdzieva A (2005) Pigments and citrinin biosynthesis by fungi belonging to genus Monascus. Z Naturforsch C 60: 116-120.

20. Xu BJ, Jia XQ, Gu LJ, Sung CK (2006) Review on the qualitative and quantitative analysis of the mycotoxin citrinin. Food Control 17: 271-285.

21. Köppen R, Koch M, Siegel D, Merkel S, Maul R, et al. (2010) Determination of mycotoxins in foods: current state of analytical methods and limitations. App Microbiol Biotechnol 86: 1595-1612.

22. Trivedi AB, Hirota M, Kitabatake N (1993) Formation of a new toxic compound citrinin $\mathrm{H} 1$, from citrinin on mild heating in water. Journal of the Chemical Society Perkin Transactions 1: 2167-2171.

23. Clark BR, Capon RJ, Lacey E, Tennant S, Gill JH (2006) Citrinin revisited: from monomers to dimers and beyond. Org Biomol Chem 4: 1520-1528.

24. Deshpande SS (2002) Handbook of Food Toxicology. Marcel Dekker, Inc. New York, NY, USA, p. 424

25. Franco CM, Fente CA, Vazquez B, Cepeda A, Lallaoui L, et al. (1996) Simple and sensitive endemic nephropathy. Journal of Agricultural and Food Chemistry 48: 2483-2488.

26. Poupko R, Luz Z, Destro R (1997) Carbon-13 NMR of citirnin in the solid state and in solutions. Journal of Physical Chemistry A 101: 5097-5102.

27. Guangmin Y, Fred M, Lok Y (2011) Citrinin derivatives from the soil filamentous fungus Penicillium sp. H9318. Journal of the Brazilian Chemical Society 22 33-37.

28. Wang ML, Lu CH, Xu QY, Song SY, Hu ZY, et al. (2013) Four new citrinin derivatives from a marine-derived Penicillium sp. fungal strain. Molecules 18 : 5723-5735.

29. O'Neil MJ (2001) The Merck Index - An encyclopedia of chemicals, drugs, and biologicals.13th Edition, Whitehouse Station, NJ: Merck and Co., Inc. p. 406. 
Citation: Doughari JH (2015) The Occurrence, Properties and Significance of Citrinin Mycotoxin. J Plant Pathol Microbiol 6: 321. doi:10.4172/21577471.1000321

30. Abdulaziz Q, Moustafa Y (2006) Application of Plackett-Burman factorial design to improve citrinin production in Monascus ruber batch cultures. Botanical Studies 47: 167-174

31. Rasmussen RR, Storm IM, Rasmussen PH, Smedsgaard J, Nielsen KF (2010) Multi-mycotoxin analysis of maize silage by LC-MS/MS. Anal Bioanal Chem 397: 765-776.

32. Hiroyuki H, Kensuke H, Kozo S (1987) Mechanism of antifungal action of citrinin. Agricultural and Biological Chemistry 5: 1373-1378.

33. Krejci ME, Bretz NS, Koechel DA (1996) Citrinin produces acute adverse changes in renal function and ultrastructure in pentobarbital-anesthetized dogs without concomitant reductions in [potassium]plasma. Toxicology 106: 167-177.
34. Sinha KK, Prasad G (1996) Effect of citrinin on pigment, protein and nucleic acid contents in maize seeds. Biologia Plantarum 38: 317-320.

35. Sándor G, Busch A, Watzke H, Reek J, Ványi A (1991) Subacute toxicity testing of ochratoxin A and citrinin in swine. Acta Vet Hung 39: 149-160.

36. Sharma RP (1993) Immunotoxicity of mycotoxins. J Dairy Sci 76: 892-897.

37. Krogh P (1987) Ochratoxin in foods. In P. Krogh (ed.), Mycotoxins in food Academic Press, London, United Kingdom pp. 97-110.

38. Mayer S, Engelhart S, Blome H (2007) The significance of mycotoxins in the framework of assessing workplace-related hazards. Gefahrstoffe Reinhaltung Der Luft 67: 407-417. 